\title{
New tricks from an old dog: Another synaptotoxic fragment from APP
}

Cell Research (2015) 25:1185-1186. doi:10.1038/cr.2015.125; published online 27 October 2015

In a surprising twist, a hitherto unrecognized cleavage of the amyloid precursor protein (APP) by $\eta$-secretase, followed by $\alpha$ - or $\beta$-secretase cleavage releases a novel

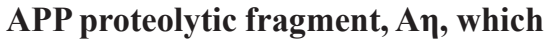
causes synaptic injury.

The phenomenon of failing to see an object even though it is right in front of our eyes is universal, though thankfully infrequent. It is usually inconsequential but when this happens in science, then the opportunity to discover something new and potentially exciting is lost. History has just repeated itself when the Haass lab reported previously unrecognized proteolytic cleavages of the amyloid precursor protein (APP) that generate fragments intermediate in size between full length APP and C-terminal fragments (CTFs), the latter being precursors to the $\mathrm{A} \beta$ peptide [1]. With the attention given to the pathways of APP processing and A $\beta$ production, it is almost shocking that this newly reported processing pathway has been uniformly ignored by the research community collectively for the past 25 years, even though the fragments are readily detected by western blotting.

Proteolytic cleavages of APP in the canonical pathway by $\alpha$ - and $\beta$-secretases generate the large soluble extracellular SAPP- $\alpha$ and sAPP- $\beta$ fragments as well as the cognate membranebound CTF- $\alpha$ and CTF- $\beta$ fragments, respectively (Figure 1). Subsequent cleavage by $\gamma$-secretase generates $A \beta$ peptide that is believed to play a seminal role in AD pathogenesis [2]. In this report, Willem and colleagues investi- gated APP fragments at approximately $25 \mathrm{kDa}$ that ends at the APP C-terminus [1]. They reasoned correctly that these fragments are processed by alternative proteases that cleave APP at a position N-terminal to the $\beta$-secretase cleavage site (Figure 1). This $\eta$ (eta)-cleavage was mapped to take place between amino acid positions 504 and 505 (APP695 numbering) and mediated in part by MT5 subclass of membrane-bound metalloproteinases (MMPs). Subsequent proteolysis by $\beta$ - or $\alpha$-secretase generates two peptides of 92 or 108 amino acids in length, designated as $A \eta-\beta$ or A $\eta-\alpha$ peptides, respectively.

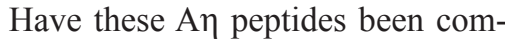
pletely relegated to the dustbin of science until their recent discovery by the Haass lab? Perhaps not because several recent studies may have detected the same cleavage or similar APP- $\eta$ fragments but incomplete characterizations in these studies were such that the fragments could not be positively identified. First is the report of two novel APP CTFs that migrate between 15 and $25 \mathrm{kDa}$, with the latter fragment serendipitously designated as $\eta-\mathrm{CTF}$ [3], but the N-terminus was not mapped or sequenced. More interesting are the studies that identified longer $A \beta$ peptides in a chinese hamster ovary $(\mathrm{CHO})$ cell line (7PA2) which overexpresses APP with the "Indiana" mutation (APPV717F) that extended upstream of $\mathrm{A} \beta$, hence designated as $\mathrm{N}$-terminally extended (NTE) A $\beta$ fragments $[4,5]$. Neither study identified A $\eta$ fragments but because NTE migrates at a molecular mass of $\sim 8-12 \mathrm{kDa}$, they might have been mistaken in the past for $A \beta$ dimers and trimers in studies that examined aggregation of low-n A $\beta$ oligomers. Unlike the Willem study, NTEs were not detected biochemically in soluble extracts from brains of AD individuals [6]. However, the physiologic significance of NTEs lie in their apparent synaptotoxicity and the increase in their levels following treatment with BACE inhibitors, precisely the same findings now reported by Willem and

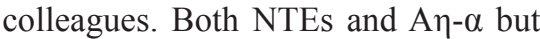
not $A \eta-\beta$ impaired synaptic plasticity as assessed by long term potentiation. Further, while $A \beta$ had been reported to induce neuronal hyperactivity, $A \eta-\alpha$, on the other hand, suppressed calcium transients. The finding that only A $\mathrm{A}-\alpha$ was synaptotoxic was not explained but curious. This is because the difference between $A \eta-\alpha$ and $A \eta-\beta$ lies only in the 16 amino acid residues between the $\beta$ - and $\alpha$-secretase cleavage sites. While $\mathrm{A} \beta$ peptide has been shown to be neurotoxic, the N-terminal portion, i.e. within $1-16$ of the $A \beta$ sequence, was reported to exhibit neurotrophic effects [2]. Indeed, earlier studies have argued that the trophic properties of sAPP are in part attributable to this same region, possibly because the -RHDS- motif at positions 5-8 exhibits cell adhesion properties in an integrin-like manner $[7,8]$. Regardless, the importance of the current finding lies in the compensatory increase

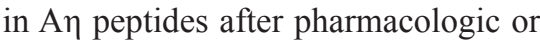
genetic inhibition of BACE activity. For example, treatment with BACE-1 inhibitor to impair $\beta$-secretase activity led to an increase in the levels of CTF- $\eta$ 


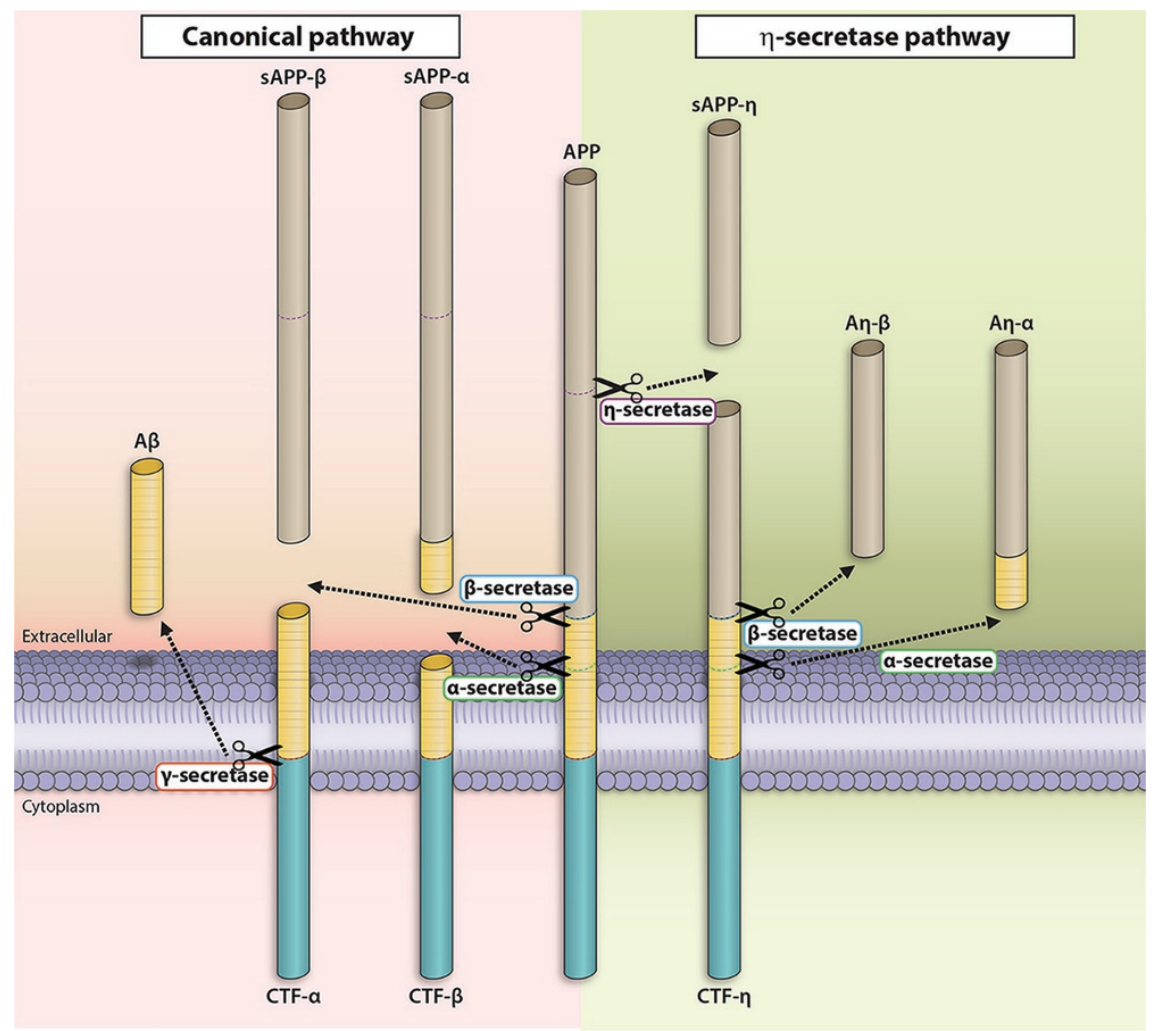

Figure 1 Schematic of APP processing pathways. The canonical pathway (left side) is represented by $\alpha$ - and $\beta$-secretase cleavages of APP near the cell surface. Sequential proteolysis by $\beta$ - and $\gamma$-secretases releases the $A \beta$ peptide. $\eta$-secretase proteolysis of APP (right side) followed by $\alpha$ - or $\beta$-secretase cleavage releases the $\mathrm{A \eta}-\alpha$ or $\mathrm{A \eta}-\beta$ peptide, respectively. (Illustrated by Karen Chiang.)

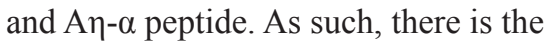
possibility of previously unrecognized toxicity of BACE inhibitors, now in the midst of late phase clinical trials testing for $\mathrm{AD}$ treatment. In this scenario, while $\mathrm{A} \beta$ production is effectively blocked by BACE inhibitors, the concurrent increase in Aך may nevertheless cause memory impairment or compromise neuronal function to offset the benefits of inhibiting $A \beta$ production. If true, this would be a nightmarish outcome for pharmaceutical companies who have spent the better part of two decades looking for small molecule inhibitors of BACE as a treatment for AD.

Evidence of physiologic $\eta$-secretase processing is supported by the presence

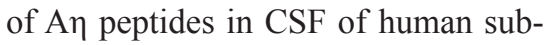
jects and in lysates of APP transgenic mice [1]. Further, levels of A $\eta$ peptides are reduced in CSF of humans with the "Swedish" APP mutation where A $\beta$ levels are increased due to enhanced $\beta$-secretase cleavage, or increased after treatment with BACE inhibitors (above), suggesting competing processing of APP by these various secretases. In brains of AD individuals and APP transgenic mice, A $\eta$ or CTF- $\eta$ epitopes were immunolocalized to dystrophic neurites, which are the distended dendrites and axons that decorate the periphery or extracellular $A \beta$ deposits. Within these neurites in APP transgenic mice, CTF- $\eta$, together with CTF- $\alpha$ and CTF- $\beta$ fragments, were detected by western blotting of tissue surrounding $A \beta$ plaque cores recovered by laser capture. Further, the presence of CTF- $\eta$ in dystrophic neurites suggested that either $\eta$-cleavage takes place at these sites, or the fragments are transported to these terminals after proteolysis elsewhere in the neuronal perikarya. Taken together, $\eta$-secretase cleavage of APP represents a constitutive processing step that is partially in dynamic equilibrium with $\alpha$ - and $\beta$-secretase-mediated proteolysis. As such, interesting follow on questions to address are where $\eta$-cleavage occurs in neurons and how this processing step is regulated, for example, is this pathway neuronal activity dependent? Finally, it will be important to resolve

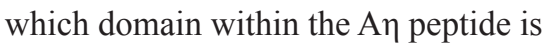
neurotoxic and whether the mechanisms of synaptic toxicity are similar or different between $A \beta$ and $A \eta$ peptides. In sum, thanks to the Haass lab, the AD field has just woken up from their collective stupor and will no doubt turn their attention to this APP processing pathway that has previously gone unnoticed right before our eyes.

Sheue-Houy Tyan ${ }^{1}$, Edward $\mathrm{H} \mathrm{Koo}^{1,2}$

${ }^{1}$ Department of Medicine, National University of Singapore, Singapore; 'Department of Neurosciences, University of California San Diego, La Jolla, USA

Correspondence: Edward H Koo

Tel: +1-858-822-1024; Fax: +1-858-822-1021

E-mail: edkoo@ucsd.edu

\section{References}

1 Willem M, Tahirovic S, Busche MA, et al. Nature 2015; 526:443-447

2 Nhan HS, Chiang K, Koo EH. Acta Neuro pathol 2015; 129:1-19.

3 Wang H, Sang N, Zhang C, et al. Biochem istry 2015; 54:2806-2816.

4 Portelius E, Olsson M, Brinkmalm G, et al. J Alzheimers Dis 2013; 33:85-93.

5 Welzel AT, Maggio JE, Shankar GM, et al. Biochemistry 2014; 53:3908-3921.

6 Mc Donald JM, O'Malley TT, Liu W, et al. Alzheimers Dement April 4 2015. pii: S1552-5260(15)00062-X.

7 Ghiso J, Rostagno A, Gardella JE, et al. Biochem J 1992; 288:1053-1059.

8 Young-Pearse TL, Chen AC, Chang R, et al. Neural Dev 2008; 3:15. 